\title{
LIE *-TRIPLE HOMOMORPHISMS INTO VON NEUMANN ALGEBRAS
}

\author{
C. ROBERT MIERS
}

\begin{abstract}
Let $M$ and $N$ be associative *-algebras. A Lie *-triple homomorphism of $M$ into $N$ is a *-linear map $\phi: M \rightarrow N$ such that

$$
\phi[[A, B], C]=[[\phi(A), \phi(B)], \phi(C)] \text {. }
$$

(Here $M$ and $N$ are considered as Lie ${ }^{*}$-algebras with $[X, Y]=X Y-Y X$.) In this note we prove that if $N$ is a von Neumann algebra with no central abelian projections and if $\phi$ is onto, there exists a central projection $D$ in $N$ such that $D \phi$ is a Lie *-homomorphism of $[M, M]$, and $(I-D) \phi$ is a Lie *antihomomorphism of $[M, M]$.
\end{abstract}

1. Introduction. An associative algebra $M$ can be turned into a Lie algebra by defining a new multiplication $[X, Y]=X Y-Y X$ where $X Y$ is the associative product of $X$ and $Y$. Every abstract Lie algebra is isomorphic to a subalgebra of a Lie algebra formed in this way. A Lie triple system is a subspace of $M$ closed under the Lie triple product $[[A, B], C]$. Lie triple systems and their homomorphisms have been studied in relation to Jordan homomorphisms of rings and the following theorem proved [2, Theorem 15]:

Let $\phi$ be a Lie triple system homomorphism of the special Lie ring $L$ and denote by $M$ the enveloping Lie ring of $\phi(L)$ and $Z$ the centre of $M$. Assume (i) $M / Z$ has no commutative Lie ideals and (ii) any two nonzero Lie ideals in $M / Z$ have nonzero intersection. Then $\phi$, when restricted to the Lie ring [ $L$, $L]$, is either a Lie homomorphism or antihomomorphism.

We wish to prove an analogous theorem when the image algebra is a von Neumann algebra. The situation is complicated by the presence, in the general case, of nonzero central projections which makes (ii) of the above theorem inapplicable.

2. Notation and preliminaries. $M$ is a *algebra over the complex field and $M_{0}, M_{1}$ subsets of $M$, then $\left[M_{0}, M_{1}\right]=$ all finite linear combinations of elements of the form $[A, B]$ with $A \in M_{0}, B \in M_{1}$. A Lie *-triple homomorphism $\phi: M \rightarrow N$ is a ${ }^{*}$-linear map preserving the Lie triple product $[[A, B]$, $C]$. The enveloping Lie algebra [2, p. 493] of $\phi(M)$ is the set $\phi(M)+[\phi(M)$, $\phi(M)$ ]. A Lie ${ }^{*}$-ideal of $M$ is a ${ }^{*}$-linear subspace $U \subseteq M$ such that if $Y \in U$, $[X, Y] \in U$ for all $X \in M$.

A von Neumann algebra $M$ is a weakly closed, selfadjoint algebra of operators on a complex Hilbert space $H$ containing the identity operator $I$. The set $Z_{M}=\{S \in M:[S, T]=0$ for all $T \in M\}$ is called the centre of $M$.

Received by the editors September 30, 1975.

AMS (MOS) subject classifications (1970). Primary 46L10; Secondary 16A68.

Key words and phrases. Lie *-triple homomorphism, von Neumann algebra. 
If $P$ is a projection (= selfadjoint idempotent) in $M$, then $M_{P}=\{P A P \mid A \in$ $M$ \}. A projection $P$ is abelian if $M_{P}$ is an abelian algebra. We use [1] as a general reference for the theory of von Neumann algebras.

The following fact will be used several times in what follows: If $M$ is a $C^{*}$-algebra, $X, Y \in M$ with $Y=Y^{*}$, then $[X, Y] \in Z_{M}$ implies $[X, Y]=0$ [2, Lemma 6]. This implies, for example, that if $M_{0}$ and $M_{1}$ are subsets of $M$ with $M_{1}$ a -subspace, then $\left[M_{0}, M_{1}\right] \subseteq Z_{M}$ implies $\left[M_{0}, M_{1}\right]=\{0\}$.

3. Lie *-triple homomorphisms. Let $\phi: M \rightarrow N$ be a Lie *-triple homomorphism where $M$ is a ${ }^{*}$-algebra over $\mathbf{C}$ and $N$ is a von Neumann algebra. The case where $N$ is a factor (that is $Z_{N}=\{\lambda I: \lambda \in \mathbf{C}\}$ ) is included separately, even though the factor case fits into the general theorem, since $\phi$ can be analyzed when $N$ is a factor by using the Jacobson-Rickart theorem already mentioned. The following result may be of independent interest.

Lemma 1. Let $N$ be a $C^{*}$-algebra. Then $N / Z_{N}$, considered as a Lie *-algebra, contains no nontrivial abelian Lie *-ideals.

Proof. Let $N_{0}$ be an abelian Lie *-ideal in $N / Z_{N}$ and let $\pi: N \rightarrow N / Z_{N}$ be the canonical Lie ${ }^{*}$-homomorphism where $\pi(A)=A+Z_{N} \cdot N_{0}$ is generated, as a *-linear space, by selfadjoint elements so let $A+Z_{N}, B+Z_{N}$ be elements of $N_{0}$ with $A-A^{*} \in Z_{N}$ and $B-B^{*} \in Z_{N}$. Then $\pi([A, B])$ $=[\pi(A), \pi(B)]=0$ since $N_{0}$ is abelian. Thus $[A, B] \in \operatorname{ker} \pi=Z_{N}$. Now $A-A^{*} \in Z_{N}$ implies $\left[A^{*}, B\right]=[A, B] \in Z_{N}$ so that $\left[A+A^{*}, B\right] \in Z_{N}$. This forces $\left[A+A^{*}, B\right]=0$. Similarly $\left[A-A^{*}, B\right]=0$. Adding, we have $[A, B]=0 . \pi^{-1}\left(N_{0}\right)+Z_{N}$ is therefore an abelian Lie *-ideal in $N$ so that by [3, Lemma 36], $\pi^{-1}\left(N_{0}\right) \subseteq Z_{N}$ or $N_{0}=\{0\}$.

THEOREM 1. If $N$ is a factor and $\phi: M \rightarrow N$ is a Lie *-triple homomorphism of $M$ onto $N$ then $\left.\phi\right|_{[M, M]}$ is a Lie *-homomorphism or a Lie *antihomomorphism.

Proof. Since $\phi$ is onto, $\phi(M)+[\phi(M), \phi(M)]=N$ so that we need only show condition (ii) of the Jacobson-Rickart theorem is fulfilled. Let $U_{1}$ and $U_{2}$ be nonzero Lie *-ideals in $N / Z_{N}$ and let $V_{1}=\pi^{-1}\left(U_{1}\right), V_{2}=\pi^{-1}\left(U_{2}\right)$. Then $V_{1}+Z_{N}, V_{2}+Z_{N}$ are Lie *-ideals in $N$ and neither is contained in $Z_{N}$.

By [3, Lemma 37] there exist nonzero two-sided ideals $g_{1}, g_{2}$ of $N$ such that $\left[q_{1}, N\right] \subseteq V_{1}+Z_{N}$ and $\left[q_{2}, N\right] \subseteq V_{2}+Z_{N}$. If $\left[q_{1}, N\right] \subseteq Z_{N}$ then $\left[q_{1}\right.$, $N]=0$ and $G_{1} \subseteq Z_{N}=\{\lambda I \mid \lambda \in \mathbf{C}\}$ which would force $g_{1}=\{0\}$. If $G_{1}=N$ then

$$
\left[q_{1}, N\right] \cap\left[q_{2}, N\right]=\left[g_{2}, N\right] \subseteq\left(V_{1}+Z_{N}\right) \cap\left(V_{2}+Z_{N}\right)
$$

so that $U_{1} \cap U_{2} \neq\{0\}$.

So we can assume $U_{1} \cap U_{2}=\{0\}$ and $\Phi_{1}, G_{2}$ are nonzero, proper ideals in $N$. Now

$$
\pi^{-1}(\{0\})=\pi^{-1}\left(U_{1} \cap U_{2}\right)=\left(V_{1}+Z_{N}\right) \cap\left(V_{2} \cap Z_{N}\right) \subseteq Z_{N} .
$$

Hence $\left[g_{1}, N\right] \cap\left[g_{2}, N\right] \subseteq Z_{N}$ which implies $\left[g_{1}, g_{2}\right] \subseteq Z_{N}$. Since $V_{1}+Z_{N}$ and $V_{2}+Z_{N}$ are selfadjoint collections, we can assume the same of $g_{1}$ and $g_{2}$ so that $\left[g_{1}, g_{2}\right]=\{0\}$. Moreover $\left[g_{1} g_{2}, N\right] \subseteq\left[g_{1}, N\right] \cap\left[g_{2}, N\right] \subseteq Z_{N}$ so that $\left[g_{1} g_{2}, N\right]=\{0\}$. Hence $g_{1} g_{2}$ is a selfadjoint two-sided ideal in $Z_{N}$ so 
that $g_{1} g_{2}=\{0\}$ which is impossible since $N$ is a factor.

We now turn our attention to the general case. As in [2] the sets

$$
N^{+}=\left\{\sum_{i=1}^{n} \phi\left[A_{i}, B_{i}\right]-\left[\phi\left(A_{i}\right), \phi\left(B_{i}\right)\right]: A_{i}, B_{i} \in M\right\}
$$

and

$$
N^{-}=\left\{\sum_{i=1}^{n} \phi\left[A_{i}, B_{i}\right]-\left[\phi\left(B_{i}\right), \phi\left(A_{i}\right)\right]: A_{i}, B_{i} \in M\right\}
$$

are Lie ideals in $\phi(M)+[\phi(M), \phi(M)]$. In our case $N^{+}$and $N^{-}$are also closed under the ${ }^{*}$-operation since $\phi$ preserves adjoints. If, for example, $N^{+} \subseteq Z_{N}$ then

$$
\begin{aligned}
0 & =[\phi[A, B]-[\phi(A), \phi(B)], \phi[X, Y]] \\
& =[\phi[A, B], \phi[X, Y]]-\phi[[A, B],[X, Y]]
\end{aligned}
$$

so that $\phi$ is a Lie ${ }^{*}$-homomorphism of $[M, M]$. Similarly, if $N^{-} \subseteq Z_{N}$ then $\phi$ is a $\mathrm{Lie}^{*}$-antihomomorphism of $[M, M]$.

Lemma 2. Let $\phi: M \rightarrow N$ be a Lie *-triple homomorphism of the *-algebra $M$ onto a von Neumann algebra $N$ which has no abelian central projections and suppose $N^{+} \nsubseteq Z_{N}$ and $N^{-} \nsubseteq Z_{N}$. There exist projections $C \neq 0$ and $D \neq 0$ in $Z_{N}$ such that $N^{+}+Z_{N} \subseteq N_{C}+Z_{N}, N^{-}+Z_{N} \subseteq N_{D}+Z_{N}$ and $C D=0$.

Proof. By [2, Theorem 14] we have $\left[N^{+}, N^{-}\right] \subseteq Z_{N}$ and so $\left[N^{+}, N^{-}\right]$ $=0$, since $N^{+}, N^{-}$are selfadjoint collections. Hence $N^{+}, N^{-}$are commuting Lie *-ideals so that $\left(N^{+}+Z_{N}\right)^{-u w}$ and $\left(N^{-}+Z_{N}\right)^{-u w}$ are also commuting Lie *-ideals. $\left(\left(N^{+}+Z_{N}\right)^{-u w}\right.$ is the ultra-weak closure of $\left(N^{+}+Z_{N}\right)$.) By [3, Theorem 4, Corollary], $\left(N^{+}+Z_{N}\right)^{-u w}=N_{C}+Z_{N},\left(N^{-}+Z_{N}\right)^{-u w}$ $=N_{D}+Z_{N}$ where $C \neq 0, D \neq 0$ are projections in $Z_{N}$. Since these Lie ${ }^{*}$-ideals commute we have $\left[N_{C}, N_{D}\right]=\left[N_{C D}, N_{C D}\right]=0$ or $C D$ is a central abelian projection. Thus $C D=0$.

THEOREM 2. Let $\phi: M \rightarrow$.N be a Lie *-triple homomorphism of a ${ }^{*}$-algebra $M$ onto a von Neumann algebra $N$ which has no central abelian projections. There exists a projection $D \in Z_{N}$ such that $D \phi$ is a Lie *-homomorphism on $[M, M]$ and $(I-D) \phi$ is a Lie *-antihomomorphism on $[M, M]$.

Proof. If $N^{+} \subseteq Z_{N}$ or $N^{-} \subseteq Z_{N}$ then $D=0$ or $D=I$. Otherwise there exist projections $C \neq 0, D \neq 0$ in $Z_{N}$ such that $N^{+}+Z_{N} \subseteq N_{C}+Z_{N}$, $N^{-}+Z_{N} \subseteq N_{D}+Z_{N}$ and $C D=0$. We have $N^{+} D=\left\{T D \mid T \in N^{+}\right\}$ $\subseteq Z_{N} D$ and $N^{-} C \subseteq Z_{N} C$. By the discussion before Lemma 2 we have that $D \phi$ is a Lie *-homomorphism of $[M, M]$ and $C \phi$ is a Lie *antihomomorphism of $[M, M]$.

Now $N^{+}(I-C-D) \subseteq Z_{N}(I-C-D)$ and $N^{-}(I-C-D)$ $\subseteq Z_{N}(I-C-D)$ so that $(I-C-D) \phi$ is both a Lie ${ }^{*}$-homomorphism and a Lie *-antihomomorphism of $[M, M]$. Thus if $X, Y \in[M, M]$,

$$
\begin{aligned}
(I-C-D) \phi[X, Y] & =(I-C-D)[\phi(X), \phi(Y)] \\
& =(I-C-D)[\phi(Y), \phi(X)] .
\end{aligned}
$$


This implies $(I-C-D) \phi(X) \phi(Y)=(I-C-D) \phi(Y) \phi(X)$ or that $(I-C-D) \phi[M, M]$ is abelian. $[[M, M], M] \subseteq[M, M]$ so that

$$
[[\phi(M), \phi(M)], \phi(M)] \subseteq \phi[M, M] .
$$

Since $\phi$ is onto, $[[N, N], N] \subseteq \phi[M, M]$ and

$$
\left[\left[N_{(I-C-D)}, N_{(I-C-D)}\right], N_{I-C-D}\right] \subseteq(I-C-D) \phi[M, M] .
$$

Hence $\left[\left[N_{(I-C-D)}, N_{I-C-D}\right], N_{I-C-D}\right]$ is an abelian Lie ${ }^{*}$-ideal in $N$ and contained in $Z_{N}$. This implies $N_{(I-C-D)}$ is abelian so that $I-C-D=0$.

REMARK 1. The requirement that $\phi$ be onto is made so that $N^{+}$and $N^{-}$, which are Lie *-ideals of $\phi(M)+[\phi(M), \phi(M)]$ will be Lie *-ideals in $N$ where a characterization of such ideals is known. Other restrictions on $M, N$ and $\phi$ can be made to insure that $\phi(M)+[\phi(M), \phi(M)]=N . \phi$ is called $L$-onto if, given $Y \in N$, there exists $X \in M$ such that $\phi(X)-Y \in Z_{N}$.

If $N$ is an infinite von Neumann allebra then $[N, N]=N[5$, Theorem 2]. Hence if $\phi$ is $L$-onto and $N$ is infinite, $[\phi(M), \phi(M)]=[N, N]=N$. If $N$ is a type I finite von Neumann algebra then $Z_{N}+[N, N]=N[4$, Theorem 1]. If in this case $\phi$ were $L$-onto and $Z_{N} \subseteq \phi(M)$, we would have $N=Z_{N}+[N$, $N] \subseteq \phi(M)+[\phi(M), \phi(M)] \subseteq N$.

REMARK 2. Modification of the arguments of [3] shows that if $M$ and $N$ are von Neumann algebras with no central abelian projections and $\phi$ is $L$-onto, then $Z_{M}$ and $Z_{N}$ are *-isomorphic.

\section{REFERENCES}

1. J. Dixmier, Les algèbres d'opérateurs dans l'espace Hilbertien, Cahiers Scientifiques, fasc. 25, Gauthier-Villars, Paris, 1969.

2. N. Jacobson and C. E. Rickart, Jordan homomorphisms of rings, Trans. Amer. Math. Soc. 69 (1950), 479-502. MR 12, 387.

3. C. R. Miers, Lie homomorphisms of operator algebras, Pacific J. Math. 38 (1971), 717-737. MR 46 \# 7918.

4. C. Pearcy and D. Topping, Commutators in certain $\mathrm{II}_{1}$-factors, J. Functional Analysis 3 (1969), 69-78. MR 39 \#89.

5. H. Sunouchi, Infinite Lie rings, Tôhoku Math. J. (2) 8 (1956), 291-307.

Department of Mathematics, University of Victoria, P. O. Box 1700, Victoria V8W 2Y2, CANADA 